\title{
Diabetic retinopathy is associated with pulse wave velocity, not with the augmentation index of pulse waveform
} Osamu Ogawa*1, Kiyoko Hiraoka², Takahiro Watanabe², Junichiro Kinoshita², Masahiko Kawasumi², Hidenori Yoshii ${ }^{2}$ and Ryuzo Kawamori²

\author{
Address: ${ }^{1}$ Midorigaoka Ekimae Clinic, 1-1-1, Midorigaoka, Yachiyo city, Chiba Pref., 276-0049, Japan and ${ }^{2}$ Department of Medicine, Metabolism \\ and Endocrinology, Juntendo University School of Medicine, 2-1-1 Hongo, Bunkyo-ku, Tokyo 113-8421, Japan \\ Email: Osamu Ogawa* - GGD03273@nifty.com; Kiyoko Hiraoka - kiyokoo@med.juntendo.ac.jp; Takahiro Watanabe - t- \\ wat@med.juntendo.ac.jp; Junichiro Kinoshita - jkinoshita@med.juntendo.ac.jp; Masahiko Kawasumi - kawasumi@med.juntendo.ac.jp; \\ Hidenori Yoshii - hdnr1967@gmail.com; Ryuzo Kawamori - kawamori@med.juntendo.ac.jp \\ * Corresponding author
}

Published: 25 April 2008

Cardiovascular Diabetology 2008, 7:II doi:10.1 I86/I475-2840-7-II
Received: 4 March 2008

Accepted: 25 April 2008

This article is available from: http://www.cardiab.com/content/7/I/II

(c) 2008 Ogawa et al; licensee BioMed Central Ltd.

This is an Open Access article distributed under the terms of the Creative Commons Attribution License (http://creativecommons.org/licenses/by/2.0), which permits unrestricted use, distribution, and reproduction in any medium, provided the original work is properly cited.

\begin{abstract}
Background: To investigate the clinical differences between pulse wave velocity and augmentation index in diabetic retinopathy.

Methods: The subjects were 201 patients with type 2 diabetes. These subjects were measured for both augmentation index (Al) and brachial-ankle pulse wave velocity (baPWV) by a pulse wave analyzer. The relationships between Al, baPWV, and diabetic retinopathy were examined.

Results: BaPWV was significantly higher in patients with diabetic retinopathy than in individuals without the disease. $(20.13 \pm 3.66 \mathrm{vs} .17 .14 \pm 3.60 \mathrm{~m} / \mathrm{s} \mathrm{p}<0.00 \mathrm{I})$ Al was higher in patients with diabetic retinopathy, but not significantly. $(19.5 \pm 15.2$ vs. $14.8 \pm 20.5 \% p=0.14)$ The association between baPWV and diabetic retinopathy remained statistically significant after adjustment. (Odds ratio: $1.21 \mathrm{Per} \mathrm{m} / \mathrm{s}$, 95\% confidence interval: $1.07-1.37$ ) On the other hand, the association between $\mathrm{Al}$ and diabetic retinopathy was not statistically significant. (Odds ratio: I.0I Per \%, 95\% confidence interval: 0.98-I.03)
\end{abstract}

Conclusion: BaPWV is associated with diabetic retinopathy, but $\mathrm{Al}$ is not. The clinical significance appears to be different between PWV and Al in patients with diabetes.

\section{Background}

Both pulse wave velocity (PWV) and augmentation index (AI) are known as markers of arterial stiffness [1]. These parameters have been associated with cardiovascular disease. The definition and measurement of these parameters is different, therefore, the clinical significance may also be different. PWV was defined as the speed with which the pulse wave travels along the length of an artery. Augmen- tation of the pulse waveform was defined as the difference between the second systolic peak (caused by wave reflection) and the first systolic peak (caused by left ventricular ejection). AI was this difference expressed as a percentage of the central pulse pressure. It is reported that type 2 diabetes is associated with increased $\mathrm{AI}$, and decreased carotid-femoral transit time, which is related to PWV [2]. On the other hand, it has been reported that there is no 
association between AI and PWV in patients with diabetes [3]. Thus, the clinical difference between PWV and AI remains uncertain in patients with diabetes. We previously reported an association between diabetic retinopathy and brachial-ankle pulse wave velocity (baPWV) [4]. However, we did not investigate the relationship between $\mathrm{AI}$ and diabetic retinopathy. In this study, to clarify the clinical difference between PWV and AI, we investigated the association between baPWV, AI and diabetic retinopathy.

\section{Methods}

Japanese patients with type 2 diabetes who had randomly undergone testing for both baPWV and AI of the common carotid artery between April 2002 and April 2003 at the outpatient department of the Juntendo University Hospital (Tokyo, Japan) were enrolled in this study. Informed consent was obtained from all subjects. Clinical data were collected in a cross sectional manner from medical records. Type 2 diabetes was indicated by a fasting plasma glucose concentration $>7.0 \mathrm{mmol} / \mathrm{L}$ without treatment, a plasma glucose concentration $11.0 \mathrm{mmol} / \mathrm{L}$ measured $2 \mathrm{~h}$ after a $75 \mathrm{~g}$ oral glucose load, or by treatment with a glucose-lowering drug. The patients selected for the study were in a state of sinus rhythm. The patients selected for the study had no history of ketoacidosis, ischemic heart disease, peripheral artery disease or ischemic stroke. Patients with an ankle-brachial pressure index $(\mathrm{ABI})<0.9$ were excluded from the analysis. Diagnoses of diabetic retinopathy were made by ophthalmologists based on the presence of one or more of the following clinical features in the fundus: hemorrhages, hard or soft exudate, venous beading, intra-retinal microvascular abnormalities, cotton-wool spots, pre-retinal new vessels, fibrous proliferation and/or photocoagulation scars. Diabetic nephropathy was defined as a casual urinary albumin/creatinine ratio $>30 \mathrm{mg} / \mathrm{g} \mathrm{Cr}$. Total cholesterol, high-density lipoprotein (HDL) cholesterol, triglyceride and hemoglobin A1c (HbA1c), urinary albumin and creatinine levels were determined using standard laboratory techniques.

\section{Measurements of PWV and AI}

Measurement of baPWV and AI were performed on the same day. The automatic pulse wave analyzer (Form/ABI with AT-unit; Omron Colin Co. Ltd, Tokyo) was used to measure baPWV, AI and ABI. This device has been approved by the US food and drug administration. The device simultaneously performs an electrocardiogram, phonocardiogram, bilateral brachial and ankle blood pressure (BP), and carotid arterial pulse wave. The detailed method to measure baPWV and ABI was previously reported $[5,6]$. Briefly, measurement was performed with the patients in the supine position after resting for five minutes. Occlusion and monitoring cuffs were placed snugly around sites in the upper and lower extremities. Pressure waveforms were then recorded simultaneously from the brachial arteries by an oscillometric method. The $\mathrm{ABI}$ was calculated as follows: $\mathrm{ABI}=$ ankle systolic $\mathrm{BP} / \mathrm{bra}-$ chial systolic BP. It is well known that patients with a low $\mathrm{ABI}$ exhibit a lower baPWV value than the actual value when assessed by the method described above [7]. Thus, we excluded patients with an ABI level of less than 0.9 when evaluating baPWV.

Carotid arterial pulse wave were obtained using arterial applanation tonometry incorporating an array of 15 micropiezoresistive transducers. Advanced multiplexing electronics combined with automatic sensor selection relative to the target artery facilitated and ensured optional placement of the sensor. The carotid sensor was manually placed on the common carotid artery. The systolic portion of the central arterial waveform is characterized by two pressure peaks. The first peak is caused by left ventricular ejection, whereas the second peak is a result of wave reflection. The difference between both pressure peaks reflects the degree to which central arterial pressure is augmented by wave reflection. The AI (\%) is defined as the percentage of the central pulse pressure which is represented by the reflected pulse wave and, therefore, reflects the degree to which central arterial pressure is augmented by wave reflection [8]. The reliability and reproducibility of the AI measured by this device are reported to be very good $[9,10]$. These measurements were taken with patients lying in a supine position after 5 min of rest.

\section{Statistical analysis}

In order to compare the association between baPWV and diabetic retinopathy, and between AI and diabetic retinopathy, we performed several statistical analyses. First, subjects were divided into two groups: those with and those without diabetic retinopathy. Clinical characteristics, including baPWV and AI, were analyzed within the two groups. Continuous variables were presented as the mean standard deviation (S.D.). Continuous variables were compared using Student's t-test and categorical data were compared using the chi-squared test. Second, we used linear regression analysis to investigate the correlation between baPWV and AI. Third, multiple regression analysis was performed to investigate the relationship between arterial stiffness markers and clinical parameters. Multiple logistic-regression analysis was used to provide the adjusted odds ratios (ORs) for baPWV and AI for diabetic retinopathy. The following factors were adjusted for: age, gender, duration of diabetes, HbA1c level, diabetic nephropathy, hypertension (systolic BP > $140 \mathrm{mmHg}$ and/or diastolic BP $>90 \mathrm{mmHg}$ ) and hyperlipidemia (total cholesterol concentration $>5.7 \mathrm{mmol} / \mathrm{L}$ and/or triglyceride concentration $>1.7 \mathrm{mmol} / \mathrm{L}$ and/or treatment with antihyperlipidemic agents). In addition, AI was 
increasing more in younger individuals $[11,12]$. Therefore, the subjects were divided into two groups, under 60 years old and over 60 years old. We analyzed relationship between AI and retinopathy each groups. The ORs are presented with 95\% confidence intervals (CIs). All statistical analyses were performed using StatView version 5.0 computer software (SAS Institute Inc. Cary, NC, USA). Probability values $(\mathrm{p})<0.05$ were considered statistically significant.

\section{Results}

The presence of diabetic retinopathy was confirmed in 47 (23.4\%) of the 201 patients who were eligible to take part in the study. Table 1 shows the characteristics of the subjects according to the presence or absence of diabetic retinopathy. BaPWV, duration of diabetes, body height, heart rate, systolic BP and total cholesterol were all significantly higher in patients with diabetic retinopathy than in individuals without the disease. The proportion of female patients, presence of nephropathy and use of antihypertensive agents were also higher among those with diabetic retinopathy. AI was higher in patients with diabetic retinopathy, but not statistically significant. On linear regression analysis, the correlation between baPWV and $\mathrm{AI}$ was weak $(\mathrm{r}=0.19, \mathrm{p}=0.007)$.

On the multiple regression analysis, age, duration, heart rate, and SBP were dependent determinants of baPWV, while age, height, heart rate, and DBP were independent determinants of AI. (table 2)

The results of the logistic regression analyses are shown in table 3. The association between baPWV and diabetic retinopathy remained statistically significant after adjustment. On the other hand, the association between AI and diabetic retinopathy was not statistically significant. Dura-
Table 2: multiple regression analysis of baPWV and Al

\begin{tabular}{lcccc}
\hline & \multicolumn{2}{c}{ BaPWV } & \multicolumn{2}{c}{ Al } \\
& $r$ & $P$ & $r$ & $P$ \\
\hline Age & 0.37 & $<0.001$ & 0.29 & $<0.001$ \\
Height & -0.04 & 0.50 & -0.31 & $<0.001$ \\
SBP & 0.50 & $<0.001$ & 0.02 & 0.86 \\
DBP & -0.16 & 0.06 & 0.26 & 0.01 \\
Heart rate & 0.25 & $<0.001$ & -0.40 & $<0.001$ \\
Duration & 0.12 & 0.02 & -0.03 & 0.61 \\
HbAlc & 0.02 & 0.74 & -0.05 & 0.41 \\
\hline
\end{tabular}

Correlation coefficients of multiple regression $(r)$ and the level of significance are shown.

tion, diabetic nephropathy were also significantly associated with diabetic retinopathy after adjustment. ORs of AI in group over 60 years old was 1.00 (95\%CI 0.97-1.04), odds ratio of AI in group under 60 years old was 1.00 (95\%CI 0.97-1.05) after adjusted age, gender, duration of diabetes, HbA1c, hypertension, hyperlipidemia, nephropathy.

\section{Discussion}

In this study, there was a significant positive association between the presence of diabetic retinopathy and baPWV among Japanese type 2 diabetic patients without macroangiopathy. However, the association between AI and diabetic retinopathy was found to be uncertain. The correlation between baPWV and AI was weak. The determining factors were different between baPWV and AI.

Previously, we showed baPWV is associated with diabetic retinopathy [4]. PWV is correlated with the duration of diabetes and with the accumulation of fluorescent advanced glycation end products in the aorta[13]. The accumulation of advanced glycation end products has

Table I: Characteristics of the 20 I patients enrolled in the study according to the presence (+) or absence (-) of diabetic retinopathy.

\begin{tabular}{llll}
\hline & $D R(+) n=47$ & DR(-) $n=154$ & $P$ value \\
\hline baPWV (m/s) & $20.13 \pm 3.66$ & $17.14 \pm 3.60$ & $<0.001$ \\
Al $(\%)$ & $19.5 \pm 15.2$ & $14.8 \pm 20.4$ & 0.14 \\
Height (m) & $1.59 \pm 0.08$ & $1.63 \pm 0.08$ & 0.003 \\
Age (years) & $61.1 \pm 10.6$ & $59.7 \pm 11.2$ & 0.44 \\
Men (n) & $25(53 \%)$ & $110(71 \%)$ & 0.03 \\
Duration (years) & $13.0 \pm 8.5$ & $8.6 \pm 7.5$ & $<0.001$ \\
Systolic BP (mmHg) & $142.7 \pm 16.3$ & $129.3 \pm 17.9$ & $<0.001$ \\
Diastolic BP (mmHg) & $82.8 \pm 11.1$ & 0.007 \\
Heart rate (beat/min) & $72.4 \pm 12.0$ & $78.3 \pm 9.56$ & 0.001 \\
HbAlc (\%) & $7.2 \pm 1.5$ & $66.3 \pm 10.5$ & 0.56 \\
Total cholesterol (mmol/L) & $5.88 \pm 2.74$ & $7.4 \pm 1.9$ & 0.004 \\
HDL cholesterol (mmol/L) & $1.40 \pm 0.47$ & $5.14 \pm 0.89$ & 0.22 \\
Triglycerides (mmol/L) & $1.68 \pm 0.88$ & $1.31 \pm 0.40$ & 0.75 \\
Nephropathy & $27(18 \%)$ & $1.74 \pm 1.12$ & $<0.001$ \\
Antihypertensive agents (yes/no) & $129 / 150$ & $27(57 \%)$ & $<0.001$
\end{tabular}

Mean values are indicated \pm the standard deviation. DR, diabetic retinopathy. 
Table 3: Adjusted odds ratios for diabetic retinopathy

\begin{tabular}{|c|c|c|c|c|c|}
\hline Independent variable & & Adjusted odds ratio $(95 \% \mathrm{Cl})$ & $P$ & Adjusted odds ratio $(95 \% \mathrm{Cl})$ & $P$ \\
\hline Age & Per year & $0.95(0.91-0.99)$ & 0.02 & $0.97(0.94-1.05)$ & 0.21 \\
\hline \multirow[t]{2}{*}{ Sex } & Female & 1.00 & & 1.00 & \\
\hline & Male & $0.33(0.14-0.78)$ & 0.01 & $0.37(0.16-0.88)$ & 0.02 \\
\hline Duration of diabetes & (per year) & $1.06(1.004-1.11)$ & 0.03 & $1.07(1.02-1.12)$ & 0.01 \\
\hline $\mathrm{HbAlc}$ & Per \% & $0.96(0.75-1.21)$ & 0.7 & $0.97(0.77-1.21)$ & 0.75 \\
\hline \multirow[t]{2}{*}{ Hypertension } & No & 1.00 & & 1.00 & \\
\hline & Yes & $1.37(0.59-3.19)$ & 0.47 & $1.91(0.86-4.28)$ & 0.11 \\
\hline \multirow[t]{2}{*}{ Hyperlipidemia } & no & 1.00 & & 1.00 & \\
\hline & yes & $0.96(0.42-2.18)$ & 0.91 & $0.90(0.4 \mathrm{I}-1.99)$ & 0.80 \\
\hline \multirow[t]{2}{*}{ Diabetic nephropathy } & No & 1.00 & & 1.00 & \\
\hline & yes & $4.57(2.04-10.28)$ & $<0.001$ & $5.24(2.40-11.4)$ & $<0.001$ \\
\hline baPWV & Per m/s & $1.21(1.07-1.37)$ & 0.002 & - & \\
\hline $\mathrm{Al}$ & Per \% & - & & $1.01(0.98-1.03)$ & 0.65 \\
\hline
\end{tabular}

Diabetic retinopathy was used as dependent variable., $\mathrm{Cl}$ : confidence interval

also been associated with the progression of diabetic retinopathy $[14,15]$. Furthermore, the United Kingdom Prospective Diabetes Study (UKPDS) showed that the development of retinopathy was associated with longterm glycemic exposure [16,17]. These findings indicate that the common mechanism of arterial stiffness and retinopathy might be the accumulation of advanced glycation end products due to chronic glycemic exposure. The result of the association of other factors and diabetic retinopathy found in this study was similar with that which we have previously reported. HbA1c and hypertension were not significantly associated with diabetic retinopathy. UKPDS showed the development of retinopathy to be associated with long-term glycemic exposure and hypertension [16-18]. The duration of diabetes was found to be associated with diabetic retinopathy in this study. However, HbA1c is a marker for short-term glycemic control ( 1 or 2 months) only; thus the effects of glycemic control on diabetic retinopathy might, therefore, not be evident in this study. In addition, BP was not measured before the onset of diabetic retinopathy in our study, so the effects of hypertension might not be apparent. These results could be a consequence of the cross-sectional study design.

The duration of diabetes determined baPWV, but not AI, in this study. In addition, PWV was increased in patients with diabetes, but this is not associated with $\mathrm{AI}$ [3]. In this study, the correlation between baPWV and AI was weak. These results indicated that chronic hyperglycemia maynot be associated with increasing AI. On the other hand, diabetic retinopathy is associated with chronic hyperglycemia. Therefore, the result that AI was not associated with diabetic retinopathy in this study may be due to AI not being associated with the duration of diabetes.

AI was correlated with height, heart rate, SBP. On the other hand, baPWV was correlated with age, SBP, and heart rate [10]. In addition, AI was influenced by vasoactive drugs independently of PWV [19]. Thus, baPWV and $\mathrm{AI}$ were not interchangeable as markers of arterial stiffness. AI has stronger potential for use in endothelial function testing rather than as a marker of arterial stiffness compared with PWV [1]. These differences between PWV and AI may have includend the results of the study.

Insulin sensitivity reportedly influences AI [20], but was not examined in this study. Differences in insulin sensitivity may influenced the result of this study. PWV and AI are both strongly related to age, but the age-related changes in $\mathrm{PWV}$ and AI are non linear, with AI increasing more in younger individuals $[11,12]$. The age of the patients in study was not as advanced. These factors may be affected the result of our study.

A positive association between AI and diabetic retinopathy has been previously reported [21]. In that study, subjects with macroangiopathy were included. Diabetic retinopathy is also reportedly associated with cardiovascular disease [22]. Both AI and PWV are also associated with macroangiopathy $[23,24]$. These associations may have influenced the reported result. In this study, to avoid the association between arterial stiffness and macroangiopathy, patients with macroangiopathy were excluded. In addition, data of height and heart rate were not included. Height and heart rate are reportedly associated with PWV and AI [10], so these unincorporated data may have influenced their result. Another possibility is that the devices in each study were different. A well-designed study will be needed to clearly elucidate the association between $\mathrm{AI}$ and diabetic retinopathy, and to identify the relevant features with utility for clinical practice. 


\section{Conclusion}

PWV and AI are reported markers of arterial stiffness, but differing results have been obtained for diabetic retinopathy. The clinical significance of PWV and AI appears to be different in patients with diabetes.

\section{Competing interests}

Omron Colin Corporation supported this study by supplying the pulse wave analyzer.

\section{Authors' contributions}

OO participated in the design of the study and drafted the manuscript. KH and TW carried out measurements of pulse wave velocity and augmentation index. JK performed statistical analyses. MK and HY contributed to the study design and revised the manuscript. RK participated in the study design and coordination. All authors read and approved the final manuscript.

\section{Acknowledgements}

The authors thank to Hirotaka Watada for helpful advice.

\section{References}

I. Oliver J], Webb DJ: Noninvasive assessment of arterial stiffness and risk of atherosclerotic events. Arterioscler Thromb Vasc Biol 2003, 23:554-566.

2. Schram MT, Henry RM, van Dijk RA, Kostense PJ, Dekker JM, Nijpels G, Heine RJ, Bouter LM, Westerhof N, Stehouwer CD: Increased central artery stiffness in impaired glucose metabolism and type 2 diabetes: the Hoorn Study. Hypertension 2004, 43:176-181

3. Lacy PS, O'Brien DG, Stanley AG, Dewar MM, Swales PP, Williams B: Increased pulse wave velocity is not associated with elevated augmentation index in patients with diabetes. J Hypertens 2004, 22:1937-1944.

4. Ogawa O, Hayashi C, Nakaniwa T, Tanaka Y, Kawamori R: Arterial stiffness is associated with diabetic retinopathy in type 2 diabetes. Diabetes Res Clin Pract 2005, 68:162-166.

5. Yamashina A, Tomiyama H, Takeda K, Tsuda H, Arai T, Hirose K, Koji Y, Hori S, Yamamoto Y: Validity, reproducibility, and clinical significance of noninvasive brachial-ankle pulse wave velocity measurement. Hypertens Res 2002, 25:359-364.

6. Tomiyama H, Koji Y, Yambe M, Motobe K, Shiina K, Gulnisa Z Yamamoto $Y$, Yamashina $A$ : Elevated $C$-reactive protein augments increased arterial stiffness in subjects with the metabolic syndrome. Hypertension 2005, 45:997-1003.

7. Suzuki E, Kashiwagi A, Nishio Y, Egawa K, Shimizu S, Maegawa H, Haneda M, Yasuda H, Morikawa S, Inubushi T, Kikkawa R: Increased arterial wall stiffness limits flow volume in the lower extremities in type 2 diabetic patients. Diabetes Care 200I, 24:2107-2||4.

8. O'Rourke MF, Gallagher DE: Pulse wave analysis. J Hypertens Suppl 1996, I 4:SI47-57.

9. Cortez-Cooper MY, Supak JA, Tanaka H: A new device for automatic measurements of arterial stiffness and ankle-brachia index. Am J Cardiol 2003, $91:$ : 519-22, A9.

10. Matsui Y, Kario K, Ishikawa J, Eguchi K, Hoshide S, Shimada K: Reproducibility of arterial stiffness indices (pulse wave velocity and augmentation index) simultaneously assessed by automated pulse wave analysis and their associated risk factors in essential hypertensive patients. Hypertens Res 2004, 27:85I-857.

I I. McEniery CM, Yasmin, Hall IR, Qasem A, Wilkinson IB, Cockcroft JR: Normal vascular aging: differential effects on wave reflection and aortic pulse wave velocity: the Anglo-Cardiff Collaborative Trial (ACCT). J Am Coll Cardiol 2005, 46: I753-I760.

12. Kohara K, Tabara Y, Oshiumi A, Miyawaki Y, Kobayashi T, Miki T: Radial augmentation index: a useful and easily obtainable parameter for vascular aging. Am J Hypertens 2005, I 8: I IS-I4S.
13. Airaksinen KE, Salmela PI, Linnaluoto MK, Ikaheimo MJ, Ahola K, Ryhanen LJ: Diminished arterial elasticity in diabetes: association with fluorescent advanced glycosylation end products in collagen. Cardiovasc Res 1993, 27:942-945.

14. Murata T, Nagai R, Ishibashi T, Inomuta H, Ikeda K, Horiuchi S: The relationship between accumulation of advanced glycation end products and expression of vascular endothelial growth factor in human diabetic retinas. Diabetologia 1997, 40:764-769.

I5. Singh R, Barden A, Mori T, Beilin L: Advanced glycation end-products: a review. Diabetologia 200I, 44:129-146.

16. Intensive blood-glucose control with sulphonylureas or insulin compared with conventional treatment and risk of complications in patients with type 2 diabetes (UKPDS 33). UK Prospective Diabetes Study (UKPDS) Group. Lancet 1998 , 352:837-853.

17. Stratton IM, Kohner EM, Aldington SJ, Turner RC, Holman RR, Manley SE, Matthews DR: UKPDS 50: risk factors for incidence and progression of retinopathy in Type II diabetes over 6 years from diagnosis. Diabetologia 2001, 44:156-I63.

18. Tight blood pressure control and risk of macrovascular and microvascular complications in type 2 diabetes: UKPDS 38. UK Prospective Diabetes Study Group. Bmj 1998 , 317:703-7|3.

19. Kelly RP, Millasseau SC, Ritter JM, Chowienczyk PJ: Vasoactive drugs influence aortic augmentation index independently of pulse-wave velocity in healthy men. Hypertension 200I, 37:|429-|433.

20. Tamminen M, Westerbacka J, Vehkavaara S, Yki-Jarvinen H: Insulininduced decreases in aortic wave reflection and central systolic pressure are impaired in type $\mathbf{2}$ diabetes. Diabetes Care 2002, 25:2314-2319.

21. Rema M, Mohan V, Deepa R, Ravikumar R: Association of carotid intima-media thickness and arterial stiffness with diabetic retinopathy: the Chennai Urban Rural Epidemiology Study (CURES-2). Diabetes Care 2004, 27:1962-1967.

22. Juutilainen A, Lehto S, Ronnemaa T, Pyorala K, Laakso M: Retinopathy predicts cardiovascular mortality in type $\mathbf{2}$ diabetic men and women. Diabetes Care 2007, 30:292-299.

23. Weber T, Auer J, O'Rourke MF, Kvas E, Lassnig E, Berent R, Eber B Arterial stiffness, wave reflections, and the risk of coronary artery disease. Circulation 2004, 109: 184-189.

24. Ogawa O, Onuma T, Kubo S, Mitsuhashi N, Muramatsu C, Kawamori $\mathrm{R}$ : Brachial-ankle pulse wave velocity and symptomatic cerebral infarction in patients with type 2 diabetes: a cross-sectional study. Cardiovasc Diabetol 2003, 2:10.
Publish with Biomed Central and every scientist can read your work free of charge

"BioMed Central will be the most significant development for disseminating the results of biomedical research in our lifetime. "

Sir Paul Nurse, Cancer Research UK

Your research papers will be:

- available free of charge to the entire biomedical community

- peer reviewed and published immediately upon acceptance

- cited in PubMed and archived on PubMed Central

- yours - you keep the copyright
BioMedcentral 